\title{
Diffusion tensor cardiovascular magnetic resonance
}

\author{
Zohya Khalique, $^{1,2}$ Dudley Pennell ${ }^{\oplus 1,2}$
}

${ }^{1}$ CMR Unit, Royal Brompton Hospital, London, UK ${ }^{2}$ National Lung and Heart Institute, Imperial College London, London, UK

\section{Correspondence to}

Professor Dudley Pennell,CMR Unit, Royal Brompton Hospital, London, London, UK; Dj.pennell@rbht.nhs.uk

Received 4 March 2019 Revised 16 April 2019 Accepted 28 April 2019 Published Online First 15 June 2019

\begin{abstract}
Cardiac structure and function are complex and interrelated. Current in vivo techniques assess the heart on a macroscopic scale, but a novel technique called diffusion tensor cardiovascular magnetic resonance (DT-CMR) can now assess the cardiac microstructure non-invasively. It provides information on the helical arrangement of cardiomyocytes that drives torsion and offers dynamic assessment of the sheetlets (aggregated cardiomyocytes) that rotate through the cardiac cycle to facilitate wall thickening. Through diffusion biomarkers, the expansion and organisation of the underlying myocardium can be described. DT-CMR has already identified novel microstructural abnormalities in cardiomyopathy, and ischaemic and congenital heart disease. This new knowledge supports the potential of DT-CMR to improve diagnostics and prognostication in various cardiac diseases.
\end{abstract}

\section{INTRODUCTION}

Cardiovascular magnetic resonance is a versatile imaging tool that offers a variety of methods of cardiac phenotyping. It is the gold standard for ventricular volumetric assessment, can assess cardiac perfusion and has the unique ability to characterise the myocardium, for example, discriminating fibrosis. ${ }^{1-3}$ Recent developments have led to an exciting new technique called diffusion tensor cardiovascular magnetic resonance (DT-CMR), which is the only method currently available to non-invasively assess the microstructure of the myocardium. Information about myocardial organisation, myocyte packing as well as cardiomyocyte and sheetlet (aggregated cardiomyocytes) orientation can be obtained. ${ }^{4-6}$ Consequently, CMR is now able to provide information on the myocardium on a scale ranging from microns to centimetres and therefore offers a deeper understanding of how the heart works in both health and disease. This review introduces DT-CMR and how preclinical and initial patient studies may shape potential future clinical application.

\section{DIFFUSION TECHNIQUE}

DT-CMR exploits the interaction between water and the myocardium. The pattern of free diffusion of water, in which molecules may diffuse out in all directions, can be depicted as a sphere. However, in heart diffusion, it is restricted by myocardial components such as cardiomyocytes, capillaries and the collagenous matrix. To assess this diffusion, images are acquired with and without diffusion weighting, which is applied through use of diffusion gradients. Weighting is described by a factor, called the $b$ value, which incorporates the gradient duration, amplitude and temporal separation of the gradient pulses. ${ }^{78}$ When diffusion weighting is applied in the presence of diffusion, signal loss occurs due to a loss in the phase coherence of spins. The measured signal attenuation is fitted on a voxel basis to derive diffusion parameter maps. For each voxel of the image, DT-CMR assumes that the restrained diffusion can be pictorially demonstrated by an ellipsoid shape and mathematically described by a tensor. ${ }^{9}$ In order to gather enough information to fill the tensor and depict the ellipsoid diffusion, it is measured in at least six different directions. The tensor gives information about how freely water can diffuse in the myocardium via mean diffusivity (MD). Typical values are in the region of $0.8-1.5 \times 10^{-3} \mathrm{~mm}^{2} / \mathrm{s}^{4}{ }^{10-16}$ The degree of diffusion restriction (how much the diffusion sphere is compressed into an ellipsoid) is described by fractional anisotropy (FA) and reflects the underlying organisation of the myocardium. FA uses a scale of 0 (highly disorganised) to 1 (extremely linearly organised). Reported values range from 0.36 to $0.61 .^{10-16}$ The diffusion ellipsoid itself has an axis system and the orientations of the principal, secondary and tertiary axes of the ellipsoid are termed E1, E2 and E3. E1 is projected on the circumferential-longitudinal plane of the left ventricle (LV) and the angle between E1 and the circumference is called E1A. E2 is projected onto the cross-myocyte plane and the angle between this and the radial direction is E2A. The orientation of the third axis of the ellipsoid is E3 and its direction is perpendicular to E2.

\section{RELATING DIFFUSION FINDINGS TO CARDIAC MICROSTRUCTURE}

E1A is also known as helix angle (HA) and has been histologically proven to align with cardiomyocyte orientation in several preclinical studies. ${ }^{461718}$ In this article, the term cardiomyocyte is preferred over myofibre or fibre to retain the distinction between cardiac and skeletal muscle. DT-CMR is capable of detecting the transmural variation in $\mathrm{HA}$, as shown in figure $1 \mathrm{~A}$. Both dissection and various histological techniques have revealed that cardiomyocytes take a left-handed helical course at the epicardium, progress through a circumferential alignment in the mesocardium and then a right-handed helix in the endocardium. ${ }^{19}{ }^{20}$ A secondary level of cardiomyocyte organisation exists. Groups of 4-12 cardiomyocytes aggregate together and are termed sheetlets. Sheetlets are surrounded by the perimysial collagen and separated by cleavage planes (figure $1 \mathrm{C}$ and G). ${ }^{2122}$ Preclinical histology work has shown that E2A aligns with sheetlet orientation. ${ }^{623} 24$ Some groups use E3 as an index of sheetlet orientation. ${ }^{25}$

To summarise, the key DT-CMR parameters are - Mean diffusivity: myocardial packing. 
- Fractional anisotropy: myocardial organisation.

- Helix angle: cardiomyocyte orientation.

- E2A: sheetlet orientation.

\section{RELATING THE MICROSTRUCTURE TO CARDIAC FUNCTION}

While histology is the current gold standard for assessing the myocardial microstructure, it is limited by being a static technique that is unable to give dynamic information about myocardial mechanics. DT-CMR can overcome this hurdle and is able to assess changes in the microstructure at various timepoints through the cardiac cycle. An in vivo, in situ, ex vivo and histology porcine study demonstrated that while there are only limited changes in HA between diastole and systole, there are significant changes in E2A through the cardiac cycle (figure 1). ${ }^{6}$ Sheetlets are aligned more parallel to the LV epicardial wall in diastole (low E2A) but reorient to a more wall-perpendicular arrangement in systole (high E2A). The degree of rotation between systole and diastole is known as 'sheetlet mobility'. This is usually about $25^{\circ}-45^{\circ} .{ }^{61526}$ The dynamic nature of sheetlets is important in explaining how cardiomyocyte thickening of only $8 \%$ translates into LV wall thickening of $40 \% .^{62027}$

The helical arrangement is also important for cardiac function. ${ }^{28} 29$ The left-handed epicardial cardiomyocytes drive clockwise rotation at the base and anticlockwise rotation at the base. The larger radius of the epicardial wall dominates the forces generated by the opposite helical arrangement of the endocardium. These opposing rotations result in torsion; the wringing motion that in conjunction with wall thickening and LV shortening aids ejection of blood from the heart in systole. The subsequent recoil and unwinding are important for passive LV filling during diastole. Thus, there are important links between the cardiac microstructure and cardiac function, which DT-CMR is helping elucidate.

\section{DT-CMR FINDINGS IN CARDIAC DISEASE Cardiomyopathy}

DT-CMR has demonstrated novel pathophysiological insight into microstructural dysfunction in patients with cardiomyopathy. In hypertrophic cardiomyopathy (HCM), diastolic E2A was significantly elevated compared with healthy controls $\left(47^{\circ}\right.$ vs $\left.24^{\circ}, \mathrm{p}<0.001\right)$, indicating that sheetlets were stuck in a predominantly systolic orientation with failure of diastolic relaxation. ${ }^{626}$ This is in keeping with the disease-causing sarcomeric mutations of HCM that result in increased myofilament sensitivity to calcium, elevated cardiomyocyte tension and impaired diastolic relaxation. Furthermore, DT-CMR may offer a non-invasive method to detect myocardial disorganisation known as disarray. ${ }^{30} 31$ This abnormality is considered a marker of HCM and has been associated with sudden cardiac death (SCD), especially in the young. ${ }^{32}$ Small studies have suggested that disarray may be detectable using the parameter FA which was reduced in the hypertrophied septum compared with the lateral wall in patients with HCM and also reduced compared with controls. ${ }^{33}{ }^{34}$ DT-CMR may potentially provide the first in vivo option to detect this histological marker that is typically identified at autopsy. Myocardial fibrosis in HCM predicts adverse outcomes including heart failure events, SCD and all-cause mortality. ${ }^{35}{ }^{36} \mathrm{MD}$ has been shown to map the extent of both focal and interstitial fibrosis and correlates with CMR measures of fibrosis in HCM. ${ }^{37}$ Together, these studies show that DT-CMR has the potential to assess the microscopic changes that occur in HCM and may help identify those patients who succumb to the adverse outcomes in this condition.
DT-CMR has also been applied in dilated cardiomyopathy (DCM). ${ }^{638}$ No significant difference was found in MD between patients with DCM and controls. However, FA was reduced in the DCM cohort, but only in diastole. ${ }^{38}$ In a different DCM study, the most striking abnormality detected was impaired sheetlet mobility. ${ }^{6}$ However, very differently to the patients with HCM, E2A was reduced in systole $\left(40^{\circ}\right.$ vs $\left.60^{\circ}, \mathrm{p}<0.001\right)$, indicating sheetlets were stuck in a more diastolic orientation and fail to reorient as expected. ${ }^{6}$ In a subsequent study, recovered patients with DCM with entirely normalised LV size and function showed residual impairment of sheetlet mobility. ${ }^{39}$ This suggests that DT-CMR may offer a role in discriminating remission from recovery in DCM.

\section{Myocardial infarction}

Structural changes occur in the acute myocardial infarct (MI) zone, where myocardial necrosis, oedema and wall thinning predominate, but over a longer period of time remote changes also occur as the LV remodels through dilatation, hypertrophy and altered ventricular architecture. ${ }^{4041}$ Several studies demonstrate increased MD and reduced FA in the acute infarct zone compared with the remote zone, in keeping with expanded disorganised ischaemic myocardium. ${ }^{42-45} \mathrm{~A}$ human study of acute infarction has shown a reduction in the right-handed cardiomyocytes that usually populate the endocardium and are the first to be affected by an ischaemic insult. ${ }^{45} \mathrm{~A}$ further longitudinal study comparing acute and chronic DT-CMR in the remote zone of patients with MI showed a correlation between the percentage increase in right-handed cardiomyocytes and the increase in wall thickening, suggesting an adaptive remodelling response. ${ }^{46}$

DT-CMR has also shown potential in delineating infarct scar by measuring a metric of 'myofibre curvature' called propagation angle (PA). ${ }^{47} 48 \mathrm{~A}$ threshold of PA greater than $4^{\circ}$ was the most accurate marker of infarct size, having the strongest correlation with gadolinium determined infarct size, as well as being associated with abnormal electroanatomic mapping voltages (figure 2). These studies indicate that DT-CMR has potential roles in managing infarction-related complications, such as predicting adverse remodelling and guiding ablation of scar-related arrhythmias.

\section{Congenital heart disease}

The helical arrangement of cardiomyocytes is typically preserved throughout mammalian species. ${ }^{19}$ However, DT-CMR has demonstrated that there is gross derangement of cardiomyocyte organisation in situs inversus totalis (SIT). ${ }^{49}$ Figure 3 shows that in SIT patients' hearts display the expected helical arrangement is inverted at the base, progresses through a mid-ventricular transition zone and becomes more like a situs solitus heart at the apex. These structural changes were associated with a significant reduction of absolute torsion in the SIT group. The transition zone wall thickening and LV shortening were also reduced compared with situs solitus hearts.

In a different report, DT-CMR has shown predominance of longitudinal and oblique fibres in the systemic right ventricle of a patient with transposition of the great arteries corrected by arterial switch. These changes were thought to be an adaptive response to the systemic pressure and load being applied to a right ventricle. ${ }^{50}$

These findings indicate that there is greater disruption to cardiac structure in congenital disease than previously appreciated and the role of the microstructure needs to be further 


\section{Helix angle}

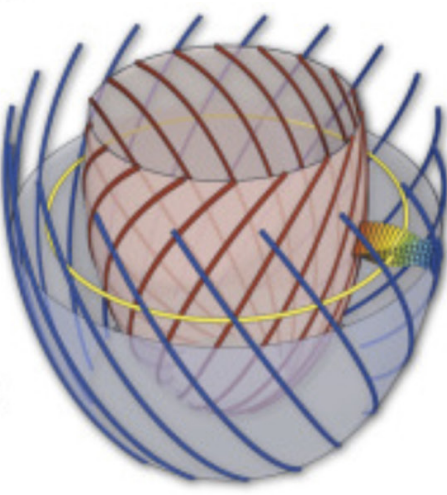

Myocyte orientation model of the LV with detail showing transmural change of helix angle
A

B

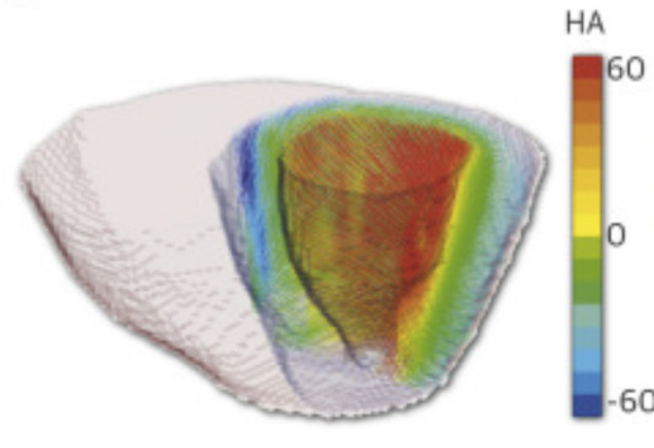

$-60$
DT-CMR tractograms color-coded according to the local E1 helix angle
B

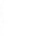

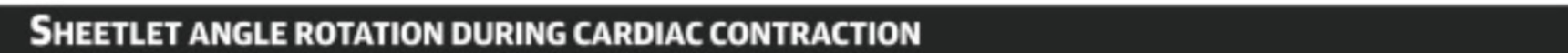

\section{Diastole}

C

D

E

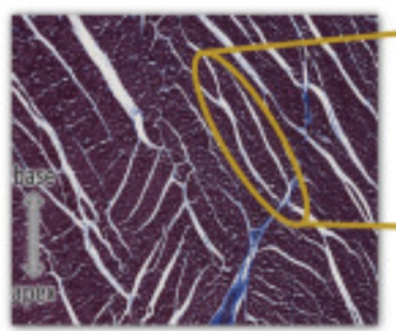

Histology image

showing sheetlets
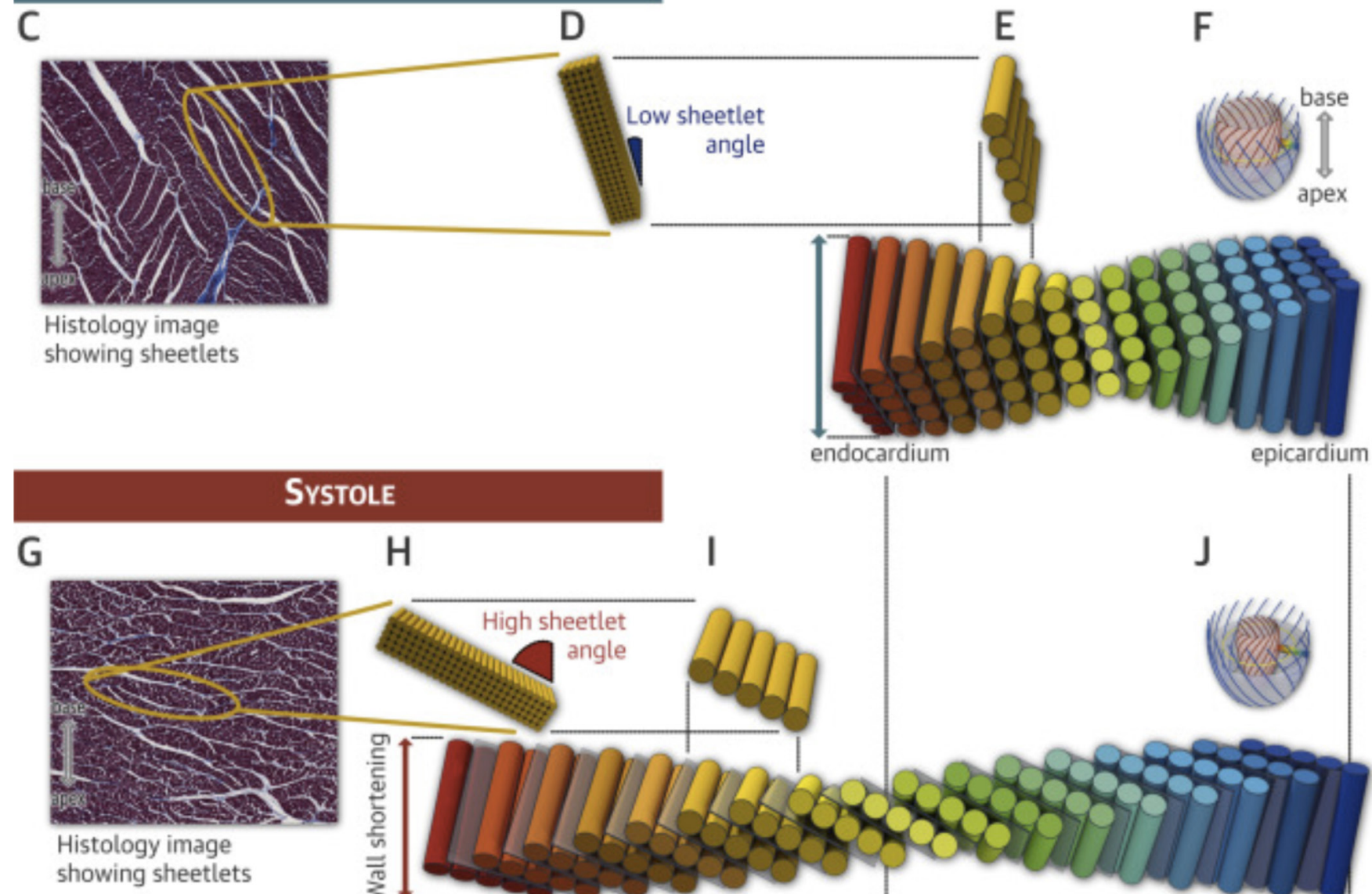

SYSTOLE

$\mathrm{H}$

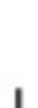

angle
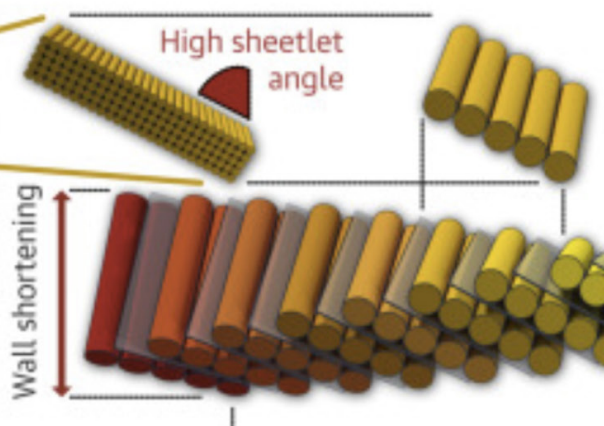

epicardium

showing sheetlets

\section{Wall thickening}

Figure 1 Heart has a primary helical organisation and secondary sheetlet structures. (A) Diffusion tensor cardiovascular magnetic resonance (DT$\mathrm{CMR}$ ) imaging of the transmural variation of helix angle (HA), where blue represents left-handed cardiomyocytes (negative helix angles), yellow are circumferential cardiomyocytes (near zero $\mathrm{HA}$ ) and red are right-handed cardiomyocytes (positive HA). A zoomed view is shown in (B). (C, G) Histology of sheetlets in diastole and systole, respectively. The orientation of the sheetlets relative to the left ventricle (LV) epicardial wall is summarised by the sheetlet angle. This is low in diastole and high in systole (C-J). At both timepoints, the helix angle (shown by the sticks, going from blue to red) does not change substantially, but there is a significant increase in sheetlet angle. Reproduced from Nielles-Vallespin et al. ${ }^{6}$ 


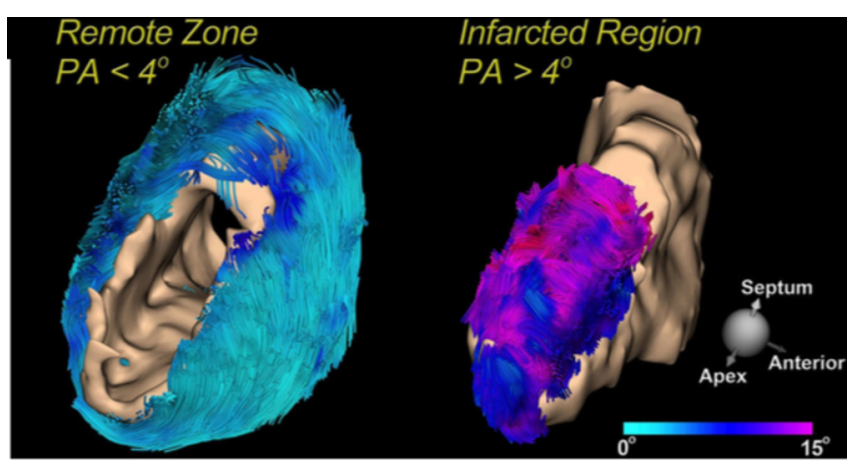

Figure 2 Diffusion tensor cardiovascular magnetic resonance (DT-CMR) can delineate myocardial infarction through use of the propagation angle (PA). DT-CMR can delineate the extent of a myocardial infarction. Areas with a PA of greater than $4^{\circ}$ correlate with infarct size, both by the CMR gold standard of late gadolinium imaging and also electroanatomical mapping. Reproduced from Mekkaoui et $a .^{48}$

investigated, particularly in the context of identifying which patients go on to develop heart failure.

\section{TECHNICAL LIMITATIONS}

DT-CMR is a novel tool that still has limitations. ${ }^{51}$ Both spatial and angular resolution could be improved. Currently, resolution is typically in the region of $2.8 \times 2.8 \times 8 \mathrm{~mm}$, affording $2-4$ voxels across a typical LV wall. ${ }^{652}$ Within each voxel, there are many thousands of cardiomyocytes, so DT-CMR cardiomyocyte and sheetlet orientations represent average values for each voxel of the images. Submillimetre diffusion tensor imaging is possible, but this is reserved for ex vivo studies. ${ }^{25} 5354$

There are two main sequence types used to obtain DT-CMR images with the key difference relating to the time over which the diffusion is measured. ${ }^{14}{ }^{16}$ A key effect of the different diffusion times is variability in FA and MD values; shorter diffusion

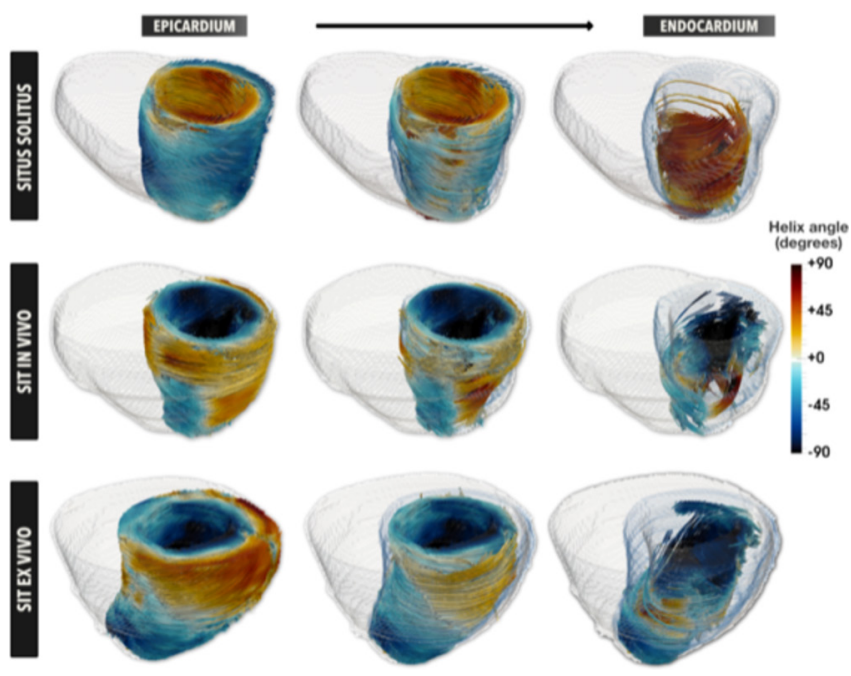

Figure 3 Helical arrangement is grossly disturbed in situs inversus totalis. The typically preserved helical arrangement of cardiomyocytes is shown in the top row. Helix angles progress from negative at the epicardium through to positive at the endocardium. In situs inversus totalis (middle and bottom rows), this pattern is inverted at the base, but then trends towards a situs solitus arrangement at the apex. There is a mid-ventricular transition zone. adapted from Khalique et al, 2018. ${ }^{39}$ times result in higher MD and lower FA values. ${ }^{14} 1655$ The spin echo technique measures diffusion over $30-40 \mathrm{~ms}$ and requires motion compensation technology, which may be less reliable in the erratic motion during diastole. ${ }^{1652} 56$ The second technique is stimulated echo acquisition mode (STEAM), and this circumvents bulk motion artefact by measuring diffusion over a whole cardiac cycle so the heart returns to its original position at the end of the diffusion period. ${ }^{57}$ The disadvantages of this approach include a strict requirement for a regular heart beat and the effects of myocardial deformation (strain) on the measured diffusion parameters. ${ }^{58}$ Compression of the myocardium during the cardiac cycle can overestimate how much diffusion has taken place and vice versa. While it is accepted that strain is a confounder, its effects are limited and ex vivo DT-CMR parameters are closer to the raw STEAM values than when strain correction algorithms are applied. ${ }^{59}$ Different scanners, scanning protocols and DT-CMR sequences exist, and there is a drive to establish standardisation between techniques and centres. Currently, scans still require approximately at least 6 breath-holds per slice. Data are typically processed post acquisition with no instant feedback on data quality, which may lead to extra images being acquired. Consequently, it may take in the region of 10-15 min for a single LV slice at both cardiac phases. As with any novel technique, there is much progress to be made to adapt DT-CMR from its current state into a mainstream clinical imaging sequence that is routinely acquired.

\section{CONCLUSION}

DT-CMR is a powerful new tool that provides microstructural information in vivo, without the need for invasive procedures or radiation exposure. Recent DT-CMR studies have improved our understanding of cardiac function in health and identified several novel abnormalities across various cardiac conditions. While not yet ready for widespread clinical use, evidence is building that

\section{Main messages}

- Diffusion tensor cardiovascular magnetic resonance is a novel non-invasive technique that assesses myocardial microstructure in vivo.

- The helical arrangement of cardiomyocytes is preserved through mammalian species but is grossly deranged in congenital heart disease.

- Sheetlet reorientation from wall-parallel to wall-perpendicular is integral to normal left ventricle wall thickening but is impaired in hypertrophic and dilated cardiomyopathy.

- Mean diffusivity and fractional anisotropy identify abnormally expanded and disorganised myocardium in hypertrophic cardiomyopathy and myocardial infarction.

\section{Current research questions}

Is there a relationship between the different genotypes and microstructural phenotypes in cardiomyopathies?

- Can diffusion tensor cardiovascular magnetic resonance (DTCMR) predict which dilated cardiomyopathy patients will recover?

- Could DT-CMR parameters identify which hypertrophic cardiomyopathy patients are at risk of sudden death?

- Can DT-CMR predict which myocardial infarction patients will adversely remodel? 
Key references

1. Streeter DD, Spotnitz HM, Patel DP, et al. Fibre Orientation in the canine left ventricle during diastole and systole. Circ Res 1969;24:339-47. doi:10.1161/01.RES.24.3.339.

2. LeGrice IJ, Smaill BH, Chai LZ, et al. Laminar structure of the heart: ventricular myocyte arrangement and connective tissue architecture in the dog. Am J Physiol-Heart Circ Physiol 1995;269:H571-82.

3. Nielles-Vallespin $\mathrm{S}$, Khalique Z, Ferreira PF, et al. Assessment of myocardial microstructural dynamics by in vivo diffusion tensor cardiac magnetic resonance. J Am Coll Cardiol 2017;69:661-76. doi:10.1016/j.jacc.2016.11.051.

4. Khalique Z, Ferreira PF, Scott $A D$, et al. Deranged myocyte microstructure in situs inversus totalis demonstrated by diffusion tensor cardiac magnetic resonance. JACC Cardiovasc Imaging 2018;11:1360-2. doi:10.1016/j.jcmg.2017.11.014.

5. Mekkaoui C, Jackowski MP, Kostis WJ, et al. Myocardial scar delineation using diffusion tensor magnetic resonance tractography. J Am Heart Assoc 2018;7:e007834. doi:10.1161/ JAHA.117.007834.

\section{Self-assessment questions}

1. Diffusion tensor cardiovascular magnetic resonance is a histologically validated technique.

2. Cardiomyocytes have a left-handed helical arrangement in the epicardium and right-handed helical arrangement in the endocardium.

3. Sheetlets are aggregated cardiomyocytes that reorient from wall-parallel in diastole to wall-perpendicular in systole.

4. Sheetlets exhibit failure of systolic relaxation in hypertrophic cardiomyopathy.

5. Mean diffusivity and fractional anisotropy both rise in the acute infarct zone.

DT-CMR shows promise in key areas such as identifying disarray pre-mortem in HCM to aid SCD risk stratification, differentiating causes of LV hypertrophy, predicting adverse outcomes such as negative remodelling and arrhythmias post MI, and discriminating patients with congenital heart disease that may go onto develop heart failure.

Contributors Both ZK and DJP wrote this review.

Funding The authors have not declared a specific grant for this research from any funding agency in the public, commercial or not-for-profit sectors.

Competing interests None declared.

Patient consent for publication Not required.

Provenance and peer review Commissioned; externally peer reviewed.

\section{REFERENCES}

1 Grothues F, Smith GC, Moon ICC, et al. Comparison of interstudy reproducibility of cardiovascular magnetic resonance with two-dimensional echocardiography in normal subjects and in patients with heart failure or left ventricular hypertrophy. Am J Cardiol 2002:90:29-34.

2 Nandalur KR, Dwamena BA, Choudhri AF, et al. Diagnostic performance of stress cardiac magnetic resonance imaging in the detection of coronary artery disease: a meta-analysis. J Am Coll Cardiol 2007;50:1343-53.

3 Kim RJ, Fieno DS, Parrish TB, et al. Relationship of MRI delayed contrast enhancement to irreversible injury, infarct age, and contractile function. Circulation 1999;100:1992-2002
4 Reese TG, Weisskoff RM, Smith RN, et al. Imaging myocardial fiber architecture in vivo with magnetic resonance. Magn Reson Med 1995;34:786-91.

5 Scollan DF, Holmes A, Winslow R, et al. Histological validation of myocardial microstructure obtained from diffusion tensor magnetic resonance imaging. Am J Physiol 1998;275:H2308-18.

6 Nielles-Vallespin S, Khalique Z, Ferreira PF, et al. Assessment of myocardial microstructural dynamics by in vivo diffusion tensor cardiac magnetic resonance. J Am Coll Cardiol 2017:69:661-76.

7 Le Bihan D, Breton E, Lallemand D, et al. MR imaging of intravoxel incoherent motions: application to diffusion and perfusion in neurologic disorders. Radiology 1986;161:401-7

8 Le Bihan D, Turner R, Moonen CT, et al. Imaging of diffusion and microcirculation with gradient sensitization: design, strategy, and significance. J Magn Reson Imaging 1991;1:7-28.

9 Basser PJ, Mattiello J, Lebihan D. Estimation of the effective self-diffusion tensor from the NMR spin echo. J Magn Reson B 1994;103:247-54.

10 Nielles-Vallespin S, Mekkaoui C, Gatehouse P, et al. In vivo diffusion tensor MRI of the human heart: reproducibility of breath-hold and navigator-based approaches. Magn Reson Med 2013:70:454-65.

11 Tunnicliffe EM, Scott AD, Ferreira P, et al. Intercentre reproducibility of cardiac apparent diffusion coefficient and fractional anisotropy in healthy volunteers. J Cardiovasc Magn Reson 2014;16.

12 Moulin K, Croisille P, Feiweier T, et al. In vivo free-breathing DTI and IVIM of the whole human heart using a real-time slice-followed SE-EPI navigator-based sequence: a reproducibility study in healthy volunteers. Magn Reson Med 2016;76:70-82.

13 McGill L-A, Scott AD, Ferreira PF, et al. Heterogeneity of fractional anisotropy and mean diffusivity measurements by in vivo diffusion tensor imaging in normal human hearts. PLoS One 2015;10:e0132360.

14 von Deuster C, Stoeck CT, Genet M, et al. Spin echo versus stimulated echo diffusion tensor imaging of the in vivo human heart. Magn Reson Med 2016;76:862-72.

15 McGill LA, Ferreira PF, Scott AD, et al. Relationship between cardiac diffusion tensor imaging parameters and anthropometrics in healthy volunteers. J Cardiovasc Magn Reson 2016;18.

16 Scott AD, Nielles-Vallespin S, Ferreira PF, et al. An in-vivo comparison of stimulatedecho and motion compensated spin-echo sequences for $3 \mathrm{~T}$ diffusion tensor cardiovascular magnetic resonance at multiple cardiac phases. J Cardiovasc Magn Reson 2018;20.

17 Hsu EW, Muzikant AL, Matulevicius SA, et al. Magnetic resonance myocardial fiber-orientation mapping with direct histological correlation. Am J Physiol 1998;274:H1627-34.

18 Holmes AA, Scollan DF, Winslow RL. Direct histological validation of diffusion tensor MRI in formaldehyde-fixed myocardium. Magn Reson Med 2000;44:157-61.

19 Pettigrew JB. On the arrangement of the muscular fibres in the ventricles of the vertebrate heart; with physiological remarks. Am J Med Sci 1866;51:497-502.

20 Streeter DD, Spotnitz HM, Patel DP, et al. Fiber orientation in the canine left ventricle during diastole and systole. Circ Res 1969;24:339-47.

21 LeGrice IJ, Smaill BH, Chai LZ, et al. Laminar structure of the heart: ventricular myocyte arrangement and connective tissue architecture in the dog. Am J Physiol 1995;269:H571-82.

22 Pope AJ, Sands GB, Smaill BH, et al. Three-dimensional transmural organization of perimysial collagen in the heart. Am J Physiol Heart Circ Physiol 2008;295:H1243-52.

23 Chen J, Liu W, Zhang $\mathrm{H}$, et al. Regional ventricular wall thickening reflects changes in cardiac fiber and sheet structure during contraction: quantification with diffusion tensor MRI. Am J Physiol Heart Circ Physiol 2005;289:H1898-H1907.

24 Kung GL, Nguyen TC, Itoh A, et al. The presence of two local myocardial sheet populations confirmed by diffusion tensor MRI and histological validation. J Magn Reson Imaging 2011;34:1080-91.

25 Hales PW, Schneider JE, Burton RAB, et al. Histo-anatomical structure of the living isolated rat heart in two contraction states assessed by diffusion tensor MRI. Prog Biophys Mol Biol 2012;110:319-30.

26 Ferreira PF, Kilner PJ, McGill L-A, et al. In vivo cardiovascular magnetic resonance diffusion tensor imaging shows evidence of abnormal myocardial laminar orientations and mobility in hypertrophic cardiomyopathy. I Cardiovasc Magn Reson 2014;16.

27 Spotnitz HM, Spotnitz WD, Cottrell TS, et al. Cellular basis for volume related wall thickness changes in the rat left ventricle. J Mol Cell Cardiol 1974;6:317-22.

28 Rüssel IK, Götte MJW, Bronzwaer JG, et al. Left ventricular torsion: an expanding role in the analysis of myocardial dysfunction. JACC Cardiovasc Imaging 2009;2:648-55.

29 Sengupta PP, Tajik AJ, Chandrasekaran K, et al. Twist mechanics of the left ventricle: principles and application. JACC Cardiovasc Imaging 2008;1:366-76.

30 Teare D. Asymmetrical hypertrophy of the heart in young adults. Heart 1958:20:1-8.

31 Maron BJ, Anan TJ, Roberts WC. Quantitative analysis of the distribution of cardiac muscle cell disorganization in the left ventricular wall of patients with hypertrophic cardiomyopathy. Circulation 1981;63:882-94.

32 Varnava AM, Elliott PM, Mahon N, et al. Relation between myocyte disarray and outcome in hypertrophic cardiomyopathy. Am J Cardiol 2001;88:275-9.

33 Ariga R, Tunnicliffe EM, Manohar SG, et al. Non-invasive imaging of myocardial disarray associates with ventricular arrhythmia in hypertrophic cardiomyopathy. Eur Heart J 2017;38(suppl_1). 
34 Tseng W-YI, Dou J, Reese TG, et al. Imaging myocardial fiber disarray and intramural strain hypokinesis in hypertrophic cardiomyopathy with MRI. J Magn Reson Imaging 2006;23:1-8.

35 Weng Z, Yao J, Chan RH, et al. Prognostic value of LGE-CMR in HCM. JACC Cardiovasc Imaging 2016;9:1392-402.

36 O'Hanlon R, Grasso A, Roughton M, et al. Prognostic significance of myocardial fibrosis in hypertrophic cardiomyopathy. J Am Coll Cardiol 2010;56:867-74.

37 Nguyen C, Lu M, Fan Z, et al. Contrast-free detection of myocardial fibrosis in hypertrophic cardiomyopathy patients with diffusion-weighted cardiovascular magnetic resonance. J Cardiovasc Magn Reson 2015;17.

38 von Deuster C, Sammut E, Asner L, et al. Studying dynamic myofiber aggregate reorientation in dilated cardiomyopathy using in vivo magnetic resonance diffusion tensor imaging. Circ Cardiovasc Imaging 2016;9:e005018.

39 Khalique Z, Ferreira PF, Scott AD, et al. Diffusion tensor cardiovascular magnetic resonance of microstructural recovery in dilated cardiomyopathy. JACC CardiovasC Imaging 2018;11:1548-50.

40 Pfeffer MA, Braunwald E. Ventricular remodeling after myocardial infarction. experimental observations and clinical implications. Circulation 1990;81:1161-72.

41 Sutton MGSJ, Sharpe N. Left ventricular remodeling after myocardial infarction. Circulation 2000;101:2981-8.

42 Chen J, Song S-K, Liu W, et al. Remodeling of cardiac fiber structure after infarction in rats quantified with diffusion tensor MRI. Am J Physiol Heart Circ Physiol 2003;285:H946-H954.

43 Nguyen C, Fan Z, Xie Y, et al. In vivo contrast free chronic myocardial infarction characterization using diffusion-weighted cardiovascular magnetic resonance. J Cardiovasc Magn Reson 2014;16.

44 Winklhofer S, Stoeck CT, Berger N, et al. Post-mortem cardiac diffusion tensor imaging: detection of myocardial infarction and remodeling of myofiber architecture. Eur Radiol 2014;24:2810-8.

45 Wu M-T, Tseng W-YI, Su M-YM, et al. Diffusion tensor magnetic resonance imaging mapping the fiber architecture remodeling in human myocardium after infarction: correlation with viability and wall motion. Circulation 2006;114:1036-45.

46 Wu M-T, Su M-YM, Huang Y-L, et al. Sequential changes of myocardial microstructure in patients postmyocardial infarction by diffusion-tensor cardiac MR: correlation with left ventricular structure and function. Circ Cardiovasc Imaging 2009;2:32-40.

47 Mekkaoui C, Jackowski MP, Kostis WJ, et al. Myocardial scar delineation using diffusion tensor magnetic resonance tractography. J Am Heart Assoc 2018;7:e007834.

48 Mekkaoui C, Huang S, Dai G, et al. Myocardial infarct delineation in vivo using diffusion tensor MRI and the tractographic propagation angle. J Cardiovasc Magn Reson 2013;15.
49 Khalique Z, Ferreira PF, Scott AD, et al. Deranged myocyte microstructure in situs inversus totalis demonstrated by diffusion tensor cardiac magnetic resonance. JACC Cardiovasc Imaging 2018;11:1360-2.

50 Harmer J, Pushparajah K, Toussaint N, et al. In vivo myofibre architecture in the systemic right ventricle. Eur Heart J 2013;34:3640.

51 Jones DK, Cercignani M. Twenty-five pitfalls in the analysis of diffusion MRI data. NMR Biomed 2010;23:803-20.

52 Stoeck CT, von Deuster C, Genet M, et al. Second-order motion-compensated spin echo diffusion tensor imaging of the human heart. Magn Reson Med 2016;75:1669-76.

53 Teh I, McClymont D, Zdora M-C, et al. Validation of diffusion tensor MRI measurements of cardiac microstructure with structure tensor synchrotron radiation imaging. J Cardiovasc Magn Reson 2017;19.

54 Helm PA, Tseng H-J, Younes L, et al. Ex vivo 3D diffusion tensor imaging and quantification of cardiac laminar structure. Magn Reson Med 2005;54:850-9.

$55 \mathrm{Kim} \mathrm{S}$, Chi-Fishman G, Barnett AS, et al. Dependence on diffusion time of apparent diffusion tensor of ex vivo calf tongue and heart. Magn Reson Med 2005;54:1387-96.

56 Gamper U, Boesiger P, Kozerke S. Diffusion imaging of the in vivo heart using spin echoes - considerations on bulk motion sensitivity. Magn Reson Med 2007:57:331-7.

57 Edelman RR, Gaa J, Wedeen VJ, et al. In vivo measurement of water diffusion in the human heart. Magn Reson Med 1994;32:423-8.

58 Reese TG, Wedeen VJ, Weisskoff RM. Measuring diffusion in the presence of material strain. J Magn Reson B 1996;112:253-8.

59 Ferreira PF, Nielles-Vallespin S, Scott AD, et al. Evaluation of the impact of strain correction on the orientation of cardiac diffusion tensors with in vivo and ex vivo porcine hearts. Magn Reson Med 2018;79:2205-15.

Answers

1. True

2. True

3. True

4. False

5. False 